\title{
Single and Double Orbital Excitations Probed by Resonant Inelastic X-ray Scattering
}

\author{
Filomena Forte, Luuk J.P. Ament and Jeroen van den Brink \\ Institute-Lorentz for Theoretical Physics, Universiteit Leiden, \\ P.O. Box 9506, 2300 RA Leiden, The Netherlands
}

(Dated: August 1, 2008)

\begin{abstract}
The dispersion of the elusive elementary excitations of orbital ordered systems, orbitions, has escaped detection so far. The recent advances in Resonant Inelastic X-ray Scattering (RIXS) techniques have made it in principle a powerful new probe of orbiton dynamics. We compute the detailed traces that orbitons leave in RIXS for an $e_{g}$ orbital ordered system, using the ultrashort core-hole life-time expansion for RIXS. We observe that both single and two orbiton excitations are allowed, with the former having sharper features, appearing lower in energy. The rich energy and momentum dependent intensity variations that we observe make clear that RIXS is an ideal method to identify and map out orbiton dispersions.
\end{abstract}

Introduction The exotic phases and phenomena exhibited by many transition metal oxides originate from the interplay of their electronic spin, charge and orbital degrees of freedom, coupled to the lattice dynamics $[1,2]$. The orbital degree of freedom, originating from the unlifted or only partially lifted local orbital degeneracy of the $3 d$ electrons, plays a particularly important role in for instance the physics of Colossal-Magneto-Resistence (CMR) manganites [3-5]. The orbitals also stand out because -in contrast to the other degrees of freedom- the dynamics of these elementary excitations is still far from being fully understood. The main reason is that it has proven very difficult to access orbital excitations experimentally.

The first claim of the observation of these elusive orbital excitations, orbitons, in $\mathrm{LaMnO}_{3}$ by optical Raman scattering [6] is very controversial [7]. Irrespective of the interpretation of these data, however, a severe limitation of the optical Raman technique is its selectiveness to excitations carrying zero momentum. This method is thus intrinsically unsuitable to map out orbiton dispersions. Other evidence for the existence of orbital excitations comes from very recent optical pump-probe experiments on manganites [8]. Even if very ingenious, also these experiments cannot provide information on the momentum dependence of orbitons.

A technique that is in principle capable of detecting the full dynamics of orbitons is Resonant Inelastic Xray Scattering (RIXS). Recently, astounding progress was made in the energy and momentum resolution of RIXS, allowing for instance the observation of magnon excitations and their dispersions in copper oxides [9]. The success of theory in describing the dispersive magnetic RIXS data [10-12] and in particular the success of the so-called ultra-short core-hole lifetime (UCL) expansion [13, 14], provides the motivation to uncover also the signatures of orbital excitations using this theoretical framework. We therefore set out to compute and predict the detailed fingerprints of the orbitons in RIXS, finding that both single and two orbiton excitations are allowed, with the former having sharper features, appearing lower in en- ergy. Orbiton scattering causes a characteristic energy and momentum dependent intensity variations in RIXS with certain selection rules. Matrix element effects also make for instance the two orbiton scattering intensity very different from the bare two-orbiton density of states. This bolsters the case that from a theoretical perspective RIXS is ideally suited to map out the orbiton dispersions $[15,16]$.

In RIXS a material is resonantly excited by tuning the energy of incoming X-rays to an atomic absorption edge, in manganites for instance the Mn K- or L-edge. At the Mn K-edge the incoming photon promotes a $1 s$ electron from the core into the $4 p$ state far above the Fermi energy, see Fig. 1. In this way a $1 s$ core-hole is created, which interacts with the $3 d$ electrons, that tend to screen the core-hole potential. After a very short time, the photoexcited electron decays, leaving the $3 d$ electrons in an excited state. From the kinetics of the outgoing X-rays, the energy and momentum of the collective excitations of the solid can be determined directly [17]. A great advantage of RIXS is that the high energy X-rays carry a momentum that is comparable to the crystal momentum in the Brioullin zone so that, unlike optical Raman experiments, RIXS can probe the full dispersion of excitations in solids. The present experimental resolution at the $\mathrm{Mn}$ edges is $\sim 100 \mathrm{meV}$. In the very near future instrumentation with an improvement with an order of magnitude in resolution at the $\mathrm{K}$ edge will become feasible [18], allowing the detection of low-lying orbital excitations.

In this Letter we determine the orbiton RIXS spectrum for an orbital ordered system with orbitals of $e_{g}$ symmetry, but the approach that we outline can equally well be used for other orbital ordering symmetries. In order to compute the RIXS spectrum it is key to determine how the intermediate state core-hole modifies the orbital dependent superexchange processes between the $3 d$ electrons. After doing so, our calculations based on the UCL expansion will show how such modifications give rise to both single and double orbiton features in the RIXS spectrum. The one-orbiton part turns out to carry most spectral weight. This is in stark contrast with 


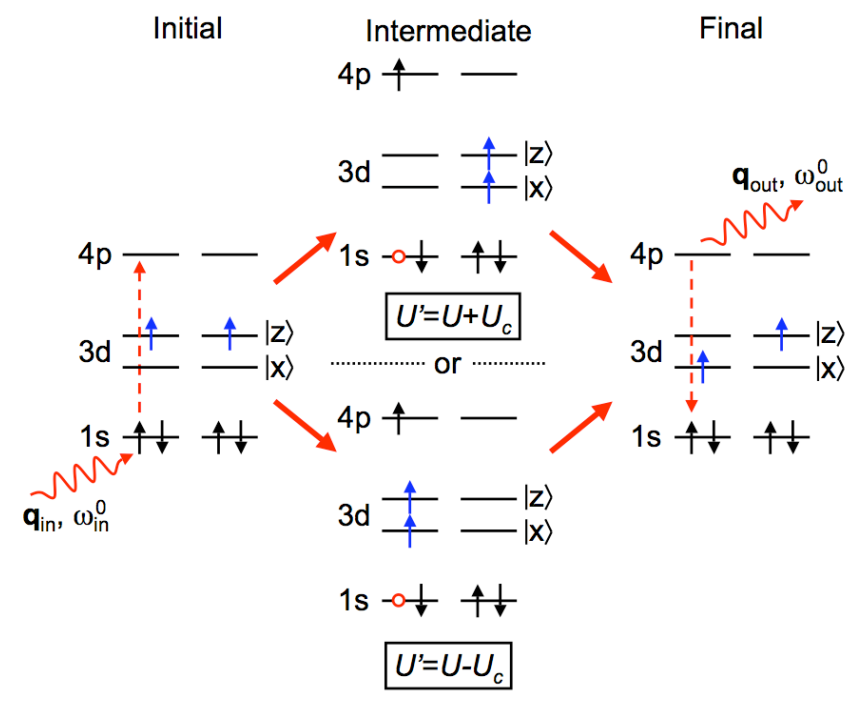

FIG. 1: The effect of the core-hole on the orbital exchange. An X-ray with energy $\omega_{\text {in }}^{0}$ and momentum $\mathbf{q}_{\text {in }}$ excites the $1 s$ electron to a $4 p$ state. Via an intermediate state, the system reaches a final state and the core-hole decays, emitting a photon of energy $\omega_{\text {out }}^{0}$ and momentum qout. In the figure the matrix element $\left\langle\downarrow_{i} \uparrow_{j}|H| \uparrow_{i} \uparrow_{j}\right\rangle$ is considered in presence of a core-hole. There are two alternative intermediate states to reach the final state. In the upper case, the amplitude is proportional to $t^{x z} t^{x x} /\left(U+U_{c}\right)$ and in the lower case to $t^{x z} t^{z z} /\left(U-U_{c}\right)$. Adding these gives a modified exchange $J^{\prime}=J(1+\eta)$ where $\eta$ depends on $U_{c}$.

magnetic RIXS, where only two-magnon scattering is allowed at zero temperature $[10,12]$. The computed orbiton spectrum for the $e_{g}$ orbital ordering of $\mathrm{LaMnO}_{3}$ shows, besides the orbiton dispersions, also strong momentum dependence of scattering intensity, with in particular a vanishing of it at $\mathbf{q}=(0,0)$ and $(\pi, \pi)$ for all but one orbiton branch. The orbiton is also expected to have phonon sidebands $[19,20]$, observable in RIXS as well.

Model Hamiltonian We focus on orbital excitations in a system with staggered $e_{g}$ orbital order, such as $\mathrm{LaMnO}_{3}$, the mother compound of the CMR manganites. The methods used below can without restriction be applied to other orbital ordered $e_{g}$ or $t_{2 g}$ systems as well, including for instance the orbital ordered titanates that are currently being investigated [21]. In the undoped manganite three $3 d$ electrons occupy the Mn $t_{2 g}$ orbitals and a fourth $3 d$ electron can be in either one of the two Mn $e_{g}$ orbitals. Below $780 \mathrm{~K}$, the $e_{g}$ orbitals order in an antiferro-orbital fashion. At lower temperatures, the spins order in an A-type magnetic structure, where the ferromagnetic, orbital ordered planes are stacked antiferromagnetically along the $c$-axis $[15,22]$.

The orbital physics of $\mathrm{LaMnO}_{3}$ can be cast in a simple pseudo-spin model, where the pseudo-spin represents the Mn $3 d e_{g}$ orbital that is occupied. It is derived starting from a generic Kondo lattice Hamiltonian [23, 24], with a local Coulomb repulsion $U$ between the electrons in the $e_{g}$ subspace. In the resulting Kugel-Khomskii model [4] the orbitals of classical antiparallel spins decouple if one neglects the Hund's rule exchange compared to the onsite Coulomb repulsion. As the quantum fluctuations associated with the large Mn core-spin on the A-type AFM structure are typically small, in leading order the orbital degrees of freedom effectively decouple along the $c$-axis, simplifying the orbital dispersion to a two-dimensional one.

The orbitals in the $a b$-plane are described by pseudospins, where pseudo-spin up corresponds to the orbital $|z\rangle \sim\left(3 r^{2}-z^{2}\right) / \sqrt{6}$ occupied and down to $|x\rangle \sim$ $\left(x^{2}-y^{2}\right) / \sqrt{2}$. The in-plane hopping integrals are $\left|t^{x x}\right|=$ $\frac{3}{4} t,\left|t^{z z}\right|=\frac{1}{4} t,\left|t^{x z}\right|=\frac{\sqrt{3}}{4} t$ with reference $t=\left|t^{z z}\right|$ along the $z$-direction. After a rotation in pseudo-spin space over an angle $\theta=\pi / 4$, the orbital model Hamiltonian is $H^{0}=\frac{J}{2} \sum_{\langle i j\rangle} H_{i j}^{0}$ with

$$
H_{i j}^{0}=3 T_{i}^{z} T_{j}^{z}+T_{i}^{x} T_{j}^{x} \pm \sqrt{3}\left(T_{i}^{z} T_{j}^{x}+T_{i}^{x} T_{j}^{z}\right)
$$

where $J=t^{2} / U[15]$. The prefactor of the $\sqrt{3}$-term is positive in the $x$-direction and negative in the $y$-direction. The classical ground state has electrons alternately occupying the orbitals $\frac{1}{\sqrt{2}}(|x\rangle+|z\rangle)$ and $\frac{1}{\sqrt{2}}(|x\rangle-|z\rangle)$. We introduce sublattice $A$ with pseudo-spin up and $B$ with pseudo-spin down and Holstein-Primakoff bosons $T_{i \in A}^{+}=$ $a_{i}, T_{i \in A}^{z}=1 / 2-a_{i}^{\dagger} a_{i}$ and $T_{j \in B}^{+}=a_{j}^{\dagger}, T_{j \in B}^{z}=a_{j}^{\dagger} a_{j}-1 / 2$. To obtain the orbital excitations we retain the terms up to quadratic order in the boson operators. After Fourier transforming the Hamiltonian and a Bogoliubov transformation, the orbiton Hamiltonian is $H^{0}=$ const. + $\sum_{\mathbf{k}} \epsilon_{\mathbf{k}} \alpha_{\mathbf{k}}^{\dagger} \alpha_{\mathbf{k}}$ with $\epsilon_{\mathbf{k}}=3 J \sqrt{1+\frac{1}{6}\left(\cos k_{x}+\cos k_{y}\right)}$. The orbiton spectrum is gapped: as our orbital Hamiltonian does not have a continuous symmetry, Goldstone modes are absent.

Modifications by core-hole In the RIXS intermediate state a core-hole is present and the Hamiltonian becomes $H=H^{0}+H^{\text {core }}$, which includes the interaction between the core-hole and the orbital degrees of freedom. The main effect of the core-hole potential is to lower the Coulomb repulsion $U$ between two $e_{g}$ electrons at the core-hole site by an amount $U_{c}$, disrupting the superexchange processes [12]. This effect is substantial as $U_{c} \approx 7 \mathrm{eV}$ [25]. To calculate the matrix elements of $H^{\text {core }}$, we consider how the core-hole changes the superexchange processes for all different pseudo-spin orientations. In Fig. 1 the two exchange paths for the specific case $\left\langle\downarrow_{i} \uparrow_{j}|H| \uparrow_{i} \uparrow_{j}\right\rangle$ are shown. The upper process involves $t^{x z} t^{x x} / U$ where $U$ is increased in presence of a core-hole with $U_{c}$. The lower process involves $t^{x z} t^{z z} / U$ and decreases the intermediate energy $U$ by $U_{c}$. These 


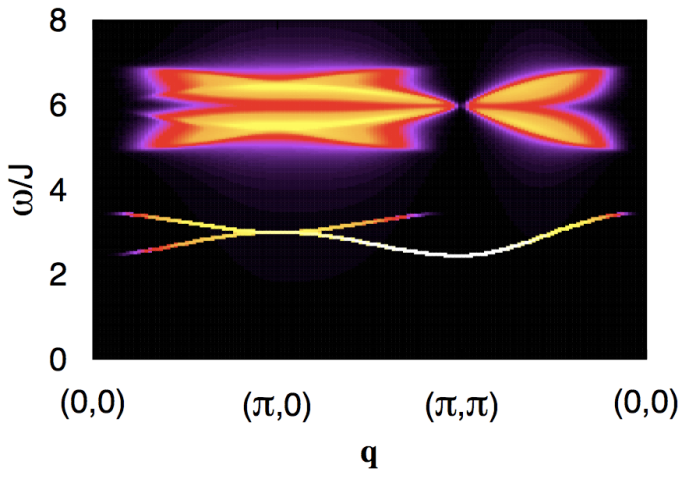

FIG. 2: The indirect RIXS spectrum for a cut through the Brillouin zone. The lower two branches originate from single orbiton excitations, the upper, continuous spectrum from double orbiton scattering. Selection rules are such that at $\mathbf{q}=(0,0)$ all spectral weight vanishes and at $\mathbf{q}=(\pi, \pi)$ only one single-orbiton branch is active.

two processes result in

$$
\begin{aligned}
\left\langle\downarrow \uparrow\left|H_{i j}^{\text {core }}\right| \uparrow \uparrow\right\rangle & =2\left(\frac{t^{x z} t^{z z}}{U-U_{c}}-\frac{t^{x z} t^{x x}}{U+U_{c}}\right)\left\langle\downarrow \uparrow\left|T_{i}^{-} T_{j}^{z}\right| \uparrow \uparrow\right\rangle \\
& = \pm \frac{\sqrt{3}}{4} J^{\prime}\left\langle\downarrow \uparrow\left|T_{i}^{-} T_{j}^{z}\right| \uparrow \uparrow\right\rangle
\end{aligned}
$$

with $J^{\prime} / J=1+\frac{U_{c}\left(U_{c}-2 U\right)}{U^{2}-U_{c}^{2}}$. Note that $J^{\prime}$ is in general different for each matrix element. Collecting the matrix elements, one finds $H=H^{0}+\frac{J}{2} \sum_{<i, j>} H_{i j}^{\text {core }} s_{i} s_{i}^{\dagger}$ with

$$
H_{i j}^{\text {core }}=\eta_{1} H_{i j}^{0}+\eta_{2}\left[\left(T_{j}^{x}-T_{i}^{x}\right) \mp \sqrt{3}\left(T_{j}^{z}-T_{i}^{z}\right)\right],
$$

where $s_{i}$ creates a core-hole and the dimensionless coupling constants are $\eta_{1}=\frac{U_{c}^{2}}{U^{2}-U_{c}^{2}}$ and $\eta_{2}=\frac{U U_{c}}{U^{2}-U_{c}^{2}}$. The ₹sign is - for bonds along the $x$-direction and + along the $y$-direction. The first term in $H_{i j}^{\text {core }}$ is similar to the one encountered in magnetic RIXS scattering. Physically it is due to the fact that the core-hole modifies the strength of the superexchange bonds to all neighboring sites. Its analytic form implies the selection rule that RIXS intensity vanishes for $\mathbf{q}=(0,0)$, as at zero momentum transfer the scattering operator is proportional to the Hamiltonian $H^{0}$ and thus commutes with it $[10,12]$. The second term, with coefficient $\eta_{2}$, contains single orbital operators and is specific for core-hole orbital coupling -in spin systems such a coupling is not allowed by conservation of $S_{\text {tot }}^{z}$. The presence of this term will allow the observation of single-orbiton scattering.

Scattering Cross section. Having derived the Hamiltonian, we can compute the RIXS spectrum using the ultrashort core-hole life-time (UCL) expansion [13, 14]. The resonant X-ray cross section for an incoming/outgoing photon with momentum $\mathbf{q}_{\text {in }} / \mathbf{q}_{\text {out }}$ and energy $\omega_{\text {in }}^{0} / \omega_{\text {out }}^{0}$ can be expressed by the Kramers-Heisenberg relation [26, 27 ] as a function of energy loss $\omega=\omega_{\text {in }}^{0}-\omega_{\text {out }}^{0}$ and momentum transfer $\mathbf{q}=\mathbf{q}_{\text {out }}-\mathbf{q}_{\text {in }}$ as $\frac{d^{2} \sigma}{d \Omega d \omega} \propto \sum_{f}\left|A_{f i}\right|^{2} \delta(\omega-$ $\left.\omega_{f i}\right)$ with

$$
A_{f i}=\omega_{\text {res }} \sum_{n} \frac{\langle f|\hat{D}| n\rangle\langle n|\hat{D}| i\rangle}{\omega_{\text {in }}-E_{n}-i \Gamma} .
$$

Here $\hat{D}$ is the dipole operator, describing the excitation from the initial state $|i\rangle$ with energy $E_{i}$ to an intermediate state $|n\rangle$ with energy $E_{n}$ and the subsequent evolution into a final state $|f\rangle$ with energy $E_{f}$, after the decay of the core-hole. The energies $E_{n}$ are measured with respect to the resonance energy $\omega_{\text {res }}$. The ultrashort core-hole life time is taken into account through an energy broadening $\Gamma$ for the intermediate state. The delta function in the cross section ensures energy conservation: the energy gained by the system $\omega_{f i}=E_{f}-E_{i}$ equals the energy lost by the photon $\omega=\omega_{\text {in }}^{0}-\omega_{\text {out }}^{0}$. The energy $\omega_{\text {in }}=\omega_{\text {in }}^{0}-\omega_{\text {res }}$ represents the detuning of the photon energy from the resonant edge [28].

When the energy of the incoming photon is tuned to the resonance $\left(\omega_{\text {in }}=0\right)$, the amplitude $A_{f i}$ can be expanded in a powerseries in $E_{n} / \Gamma[13,14]$. The zeroth order term gives only elastic scattering and is thus omitted in the following. We can retain only the lowest order terms in $\eta_{1,2} J / \Gamma \approx 0.2$ in the expansion of $H^{l}=\left(H^{0}+H^{\text {core }}\right)^{l}$. This approximation is controlled by the large core-hole broadening $(\Gamma \approx 0.58 \mathrm{eV}$ at the $\mathrm{Mn}$ K-edge, $1.3 \mathrm{eV}$ at the $\mathrm{Mn} \mathrm{L}_{1}$-edge and $0.16 \mathrm{eV}$ at the $\mathrm{Mn}$ $\mathrm{L}_{2,3}$-edges [29]) and the values of $J \approx 25 \mathrm{meV}[25,30]$ and $U_{c} / U \approx 1.1$. With this the expression for the scattering simplifies to $A_{f i}=\frac{\omega_{\text {res }}}{i \Gamma} \frac{1}{i \Gamma+\omega}\left\langle f\left|\hat{O}_{\mathbf{q}}\right| i\right\rangle$, with the effective scattering operator $\hat{O}_{\mathbf{q}}=\frac{J}{2} \sum_{<i, j>} e^{i \mathbf{q} \cdot \mathbf{R}_{i}} H_{i j}^{\text {core }}$. We evaluate this expression in terms of the boson creation and annihilation operators, in linear spinwave approximation. After Fourier transforming and introducing the Bogoliubov transformed orbiton operators, we obtain the single- and double-orbiton scattering operators, $\hat{O}_{\mathbf{q}}^{(1)}$ and $\hat{O}_{\mathbf{q}}^{(2)}=\sum_{\mathbf{k}} \hat{O}_{\mathbf{k}, \mathbf{q}}^{(2)}$, respectively. At $T=0$, the singleorbiton scattering operator is

$$
\begin{aligned}
\hat{O}_{\mathbf{q}}^{(1)}= & -\frac{\eta_{1} \sqrt{3 N}}{8} J_{\mathbf{q}}^{-}\left(u_{\overline{\mathbf{q}}}-v_{\overline{\mathbf{q}}}\right) \alpha_{-\overline{\mathbf{q}}}^{\dagger} \\
& +\frac{\eta_{2} \sqrt{N}}{4}\left(J_{\mathbf{q}}-J_{\mathbf{0}}\right)\left(u_{\mathbf{q}}-v_{\mathbf{q}}\right) \alpha_{-\mathbf{q}}^{\dagger}
\end{aligned}
$$

and the double orbiton scattering operator

$$
\begin{aligned}
\hat{O}_{\mathbf{k}, \mathbf{q}}^{(2)}= & -\frac{\eta_{1}}{8}\left[\left(6\left(J_{\mathbf{q}}+J_{\mathbf{0}}\right)+J_{\mathbf{k}}+J_{\mathbf{k}+\mathbf{q}}\right) u_{\mathbf{k}} v_{\mathbf{k}+\mathbf{q}}\right. \\
& \left.-J_{\mathbf{k}+\mathbf{q}}\left(u_{\mathbf{k}} u_{\mathbf{k}+\mathbf{q}}+v_{\mathbf{k}} v_{\mathbf{k}+\mathbf{q}}\right)\right] \alpha_{\mathbf{k}}^{\dagger} \alpha_{-\mathbf{k}-\mathbf{q}}^{\dagger} \\
& +\frac{\eta_{2} \sqrt{3}}{2} J_{\mathbf{q}}^{-} u_{\mathbf{k}} v_{\mathbf{k}+\overline{\mathbf{q}}} \alpha_{\mathbf{k}}^{\dagger} \alpha_{-\mathbf{k}-\overline{\mathbf{q}}}^{\dagger},
\end{aligned}
$$

where $u_{\mathbf{k}}$ and $v_{\mathbf{k}}$ are the coefficients of the Bogoliubov transformation, $\overline{\mathbf{q}}=\mathbf{q}+(\pi, \pi), J_{\mathbf{k}}^{x(y)}=2 J \cos k_{x(y)}, J_{\mathbf{k}}=$ $J_{\mathbf{k}}^{x}+J_{\mathbf{k}}^{y}$ and $J_{\mathbf{k}}^{-}=J_{\mathbf{k}}^{x}-J_{\mathbf{k}}^{y}$. As expected, since the $z$ component of the total pseudospin $T_{\text {tot }}^{z}=\sum_{i} T_{i}^{z}$ is not 


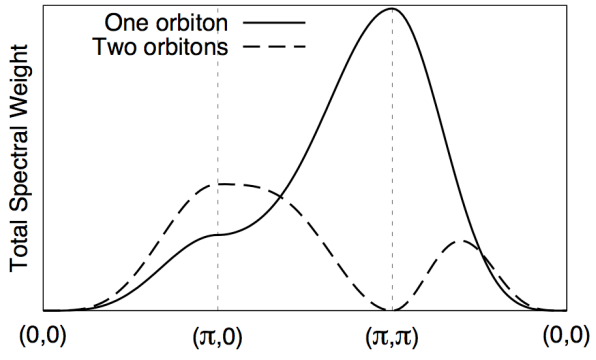

FIG. 3: Comparison of the energy integrated spectral weight of the one- and two-orbiton RIXS spectra at fixed momentum transfer $\mathbf{q}$.

conserved in the scattering process, we get a contribution to the scattering intensity both from the one- and two-orbiton part. This is a fundamental difference with respect to magnetic RIXS spectrum, where the conservation of total $\mathrm{S}^{z}$ allows only creation/annihilation of an even number of magnons [10, 12].

The resulting RIXS spectrum is shown in Fig. 2. We observe that the two-orbiton spectrum vanishes not only at $\mathbf{q}=(0,0)$ but also at the antiferro-orbital ordering wavevector $(\pi, \pi)$. This is due to the RIXS matrix elements and not to the two-orbiton DOS, which actually peaks at $(\pi, \pi)$. The total spectral weight of the orbiton spectrum is strongly q-dependent. In Fig. (3) we compare the spectral weights: the one-orbiton weight dominates and peaks at $\mathbf{q}=(\pi, \pi)$, where the two-orbiton spectrum vanishes. The two-orbiton spectrum has its maximum total weight at $(\pi, 0)$, where the total twoorbiton intensity actually outweighs the one-orbiton one. An exchange constant of $J \approx 25 \mathrm{meV}$ will put the twoorbiton spectrum around $\omega \approx 150 \mathrm{meV}$. The one-orbiton peak at $(\pi, \pi)$ is much more intense, but at $\omega \approx 2.4 J \approx 60$ meV, might be more difficult to discern from the tail of the elastic peak.

Conclusion Our calculations shown that in resonant inelastic X-ray experiments orbital excitations are distinguishable by characteristic variations in scattering amplitude as a function of both energy and momentum transfer. Both single and two orbiton excitations are allowed, with intensities that are of the same order. The single orbiton features are sharp and lower in energy, the double orbiton ones are higher in energy and more smeared out. At high symmetry points in the Brioullin zone, the intensity of specific orbiton branches vanishes. Our detailed predictions on the orbiton spectrum of an $e_{g}$ orbital ordered system bolster the case that with RIXS it will for the first time be possible to directly probe orbiton dynamics and dispersions. The necessary energy and momentum resolution starts to come within reach of experiment. An observation of orbitons in RIXS will open the way to probe new orbital related quasiparticles, for instance orbiton-magnon bound states for which so far only theoretical evidence exists [31].
Acknowledgements We thank Sumio Ishihara, Giniyat Khaliullin and Jan Zaanen for stimulating discussions.

[1] M. Imada, A. Fujimori, and Y. Tokura, Rev. Mod. Phys. 70, 1039 (2000).

[2] Y. Tokura and N. Nagaosa, Science 288, 462 (2000).

[3] B. Goodenough, Phys. Rev. 100, 564 (1955).

[4] K. I. Kugel and D. I. Khomskii, Sov. Phys. Usp. 25, 231 (1982).

[5] J. van den Brink, G. Khaliullin and D. Khomskii in Colossal Magnetoresistive Manganites, ed. T. Chatterji, Kluwer Academic Publishers, Dordrecht (2004).

[6] E. Saitoh et. al., Nature 410, 180 (2001).

[7] M. Gruninger et. al., Nature 418, 39 (2002).

[8] D. Polli et al., Nature Materials 6, 643 (2007).

[9] J.P. Hill et. al., Phys. Rev. Lett. 100, 097001 (2008).

[10] J. van den Brink, Europhysics Letters 80, 47003 (2007).

[11] T. Nagao and J. I. Igarashi, Phys. Rev. B 75, 214414 (2007).

[12] F. Forte, L.J.P. Ament and J. van den Brink, Phys. Rev. B. 77, 134428 (2008).

[13] J. van den Brink and M. Veenendaal, Europhysics Letters, 73121 (2006).

[14] L.J.P. Ament, F. Forte and J. van den Brink, Phys. Rev. B 75, 115118 (2007).

[15] J. van den Brink, P. Horsch, F. Mack and A. M. Oleś, Phys. Rev. B 59, 6795 (1999).

[16] S. Ishihara and S. Maekawa, Phys. Rev. B. 62, 2338 (2000).

[17] For a review see: A. Kotani and S. Shin, Rev. Mod. Phys. 73, 203 (2001).

[18] H. Yavas and E. Alp, private communication.

[19] J. van den Brink, Phys. Rev. Lett. 87, 217202 (2001).

[20] K.P. Schmidt, M. Gruninger and G.S. Uhrig, Phys. Rev. B. 76, 075108 (2007).

[21] C. Ulrich et al., private communication, to be published.

[22] S. Ishihara et al., New J. Phys. 7, 119 (2005).

[23] P. Horsch, J. Jaklič and F. Mack, Phys. Rev. B 59, 6217 (1999).

[24] J. van den Brink, New Journal of Physics 6, 201 (2004).

[25] S. Ishihara and S. Maekawa, Phys. Rev. Lett. 80, 3799 (1998).

[26] H.A. Kramers and W. Heisenberg, Z. Phys. 31, 681 (1925).

[27] M. Blume, J. Appl. Phys. 57, 3615 (1985).

[28] Note that the lifetime broadening only appears in the intermediate states and not in the nal or initial states as these both have very long lifetimes. This implies that the core-hole broadening does not present an intrinsic limit to the experimental resolution of RIXS: the loss energy $\omega$ is completely determined by kinematics.

[29] M. O. Krause and J. H. Oliver, J. Phys. Chem. Ref. Data 8, 329 (1979).

[30] J. van den Brink, P. Horsch and A. M. Oleś, Phys Rev. Lett. 85, 5174 (2000).

[31] J. van den Brink, W. Stekelenburg, D.I. Khomskii, G.A. Sawatzky and K.I. Kugel, Phys. Rev. B. 58, 10276 (1998). 